\title{
DỨBin
}

Technological University Dublin ARROW@TU Dublin

\section{Daemon Decay and Cosmic Inflation}

\author{
Emil Prodanov \\ Technological University Dublin, emil.prodanov@tudublin.ie
}

Follow this and additional works at: https://arrow.tudublin.ie/scschmatart

Part of the Mathematics Commons

\section{Recommended Citation}

Prodanov, E. (2011). Daemon decay and cosmic inflation. In (Klapdor-Kleingrothaus, H.V., Krivosheina, I.V.\& Viollier, R. ) (eds) Physics Beyond the Standard Model of Particles, Cosmology and Astrophysics: Proceedings of the Fifth International Conference Beyond 2010, World Scientific.

This Conference Paper is brought to you for free and open access by the School of Mathematics at ARROW@TU Dublin. It has been accepted for inclusion in Articles by an authorized administrator of ARROW@TU Dublin. For more information, please contact arrow.admin@tudublin.ie, aisling.coyne@tudublin.ie, gerard.connolly@tudublin.ie.

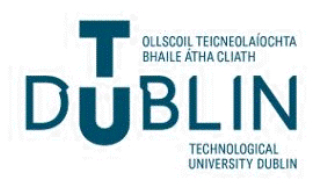




\section{PHISIES BEYOND THE}

STANDARD MODELS OF PABUTCHSS, BOSMOLOEY AND ASTIODLUESES

Proceedings of the Ffth International Conference- Bsyonil2010 a.

H V Klapdor-Kleingrothaus ?

I V Krivoshoina

PIVollier

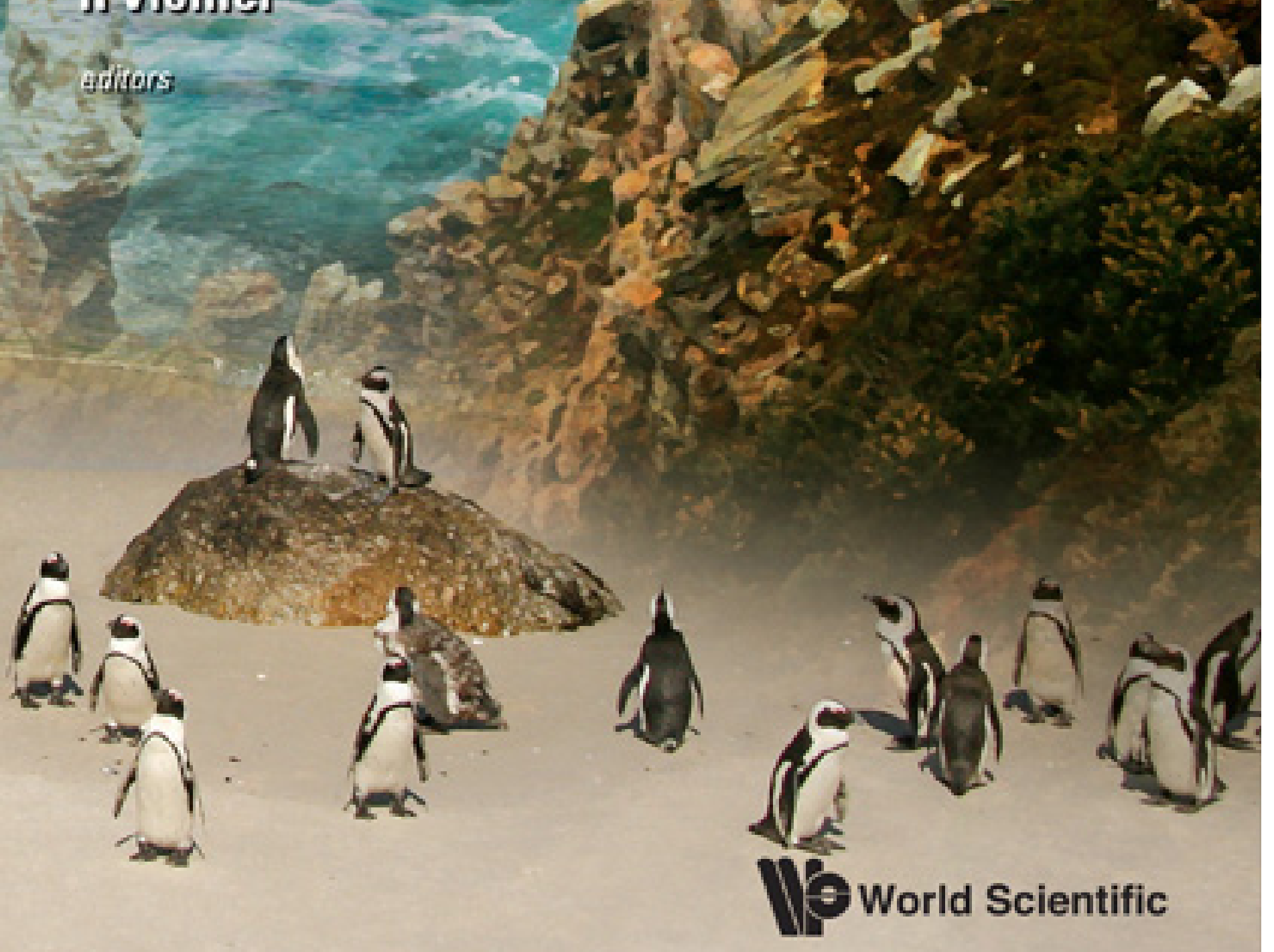




\title{
DAEMON DECAY AND COSMIC INFLATION
}

\author{
EMIL M. PRODANOV \\ School of Mathematical Sciences, Dublin Institute of Technology, Ireland \\ E-mail: emil.prodanov@dit.ie \\ www.maths.dit.ie
}

\begin{abstract}
Quantum tunneling in Reissner-Nordström geometry is studied and the tunneling rate is determined. A possible scenario for cosmic inflation, followed by reheating phases and subsequent radiation-domination expansion, is proposed.
\end{abstract}

Keywords: Early Universe, Inflation, Reissner-Nordström

In 1971, Hawking suggested ${ }^{1}$ that there may be a very large number of gravitationally collapsed charged objects of very low masses, formed as a result of fluctuations in the early Universe. A mass of $10^{14} \mathrm{~kg}$ of these objects could be accumulated at the centre of a star like the Sun. The masses of these collapsed objects are from $10^{-8} \mathrm{~kg}$ and above and their charges are up to \pm 30 electron units. ${ }^{1}$

Tracing the evolution of such objects, we propose a mechanism that accounts for the cosmic inflation, takes us into a period of reheating phases, and, finally, into the expansion of a radiation-dominated Universe. In a nut-shell, the inflation mechanism is based on the accumulative effects of Coulomb repulsion at very short range, initially completely "cocooned" by Reissner-Nordström gravitational effects and subsequently unleashed by quantum tunneling.

Consider the Reissner-Nordström geometry ${ }^{2,3}$ in Boyer-Lindquist coordinates: ${ }^{4}$

$$
d s^{2}=-\frac{\Delta}{r^{2}} d t^{2}+\frac{r^{2}}{\Delta} d r^{2}+r^{2} d \theta^{2}+r^{2} \sin ^{2} \theta d \phi^{2}
$$

where: $\Delta=r^{2}-2 M r+Q^{2}, M$ is the mass of the centre, and $Q$ - the charge of the centre. We will be interested in the case of a naked singularity only, namely: $|Q|>M$.

The radial motion of an ultra-relativistic test particle of mass $m$ and charge $q$ in Reissner-Nordström geometry can be modeled by an effective one-dimensional motion of a particle in non-relativistic mechanics with the following equation of motion $^{5-7}$ (see also ${ }^{8}$ for Schwarzschild geometry) :

$$
\frac{\dot{r}^{2}}{2}+U(r)=\frac{\epsilon^{2}-1}{2}
$$


where

$$
U(r)=\frac{1}{2}\left(1-\frac{q^{2}}{m^{2}}\right) \frac{Q^{2}}{r^{2}}-\left(1-\frac{q}{m} \frac{Q}{M} \epsilon\right) \frac{M}{r} \equiv-\frac{a}{r^{2}}+\frac{b}{r}
$$

is the effective non-relativistic one-dimensional potential per unit mass, $E=\left(\epsilon^{2}-\right.$ $1) / 2$ is the specific energy of the effective one-dimensional motion, and $\epsilon=k T / m+1$ is the specific energy of the three-dimensional relativistic motion. In equation (3), the constant $a=-Q^{2}\left(1-q^{2} / m^{2}\right) / 2$ is positive in view of the very high charge-tomass ratio $q / m$ for all charged elementary particles and the parameter $b=-M[1-$ $(q Q \epsilon) /(m M)]$ depends on the temperature via $\epsilon$. Motion is allowed only when the kinetic energy is real. Equation (2) determines the region $\left(r_{-}, r_{+}\right)$within which classical motion is impossible. The turning radii are given by: ${ }^{5-7}$

$$
r_{ \pm}=\frac{M}{\epsilon^{2}-1}\left[\epsilon \frac{q}{m} \frac{Q}{M}-1 \pm \sqrt{\left(\epsilon \frac{q}{m} \frac{Q}{M}-1\right)^{2}-\left(1-\epsilon^{2}\right)\left(1-\frac{q^{2}}{m^{2}}\right) \frac{Q^{2}}{M^{2}}}\right] .
$$

There is no inner turning radius $r_{-}$for particles of specific charge $q / m$ such that $\operatorname{sign}(Q) q / m<1$. For particles such that $\operatorname{sign}(Q) q / m<-1$, there is neither inner turning radius, nor outer turning radius. ${ }^{5-7}$ Such particles will fly unopposed into the centre. Barrier with two turning radii is present only for particles for which $\operatorname{sign}(Q) q / m \geq 1$. We will consider only such particles. Thus the parameter $b$ will be taken as positive. There is no classical analogue of this effect: a charged centre being able to capture particles of the same charge within the inner turning radius $r_{-}$, despite of the Coulomb repulsion. We make the following assumption: the preinflationary Universe is an ideal quantum gas in thermal equilibrium with constant volume densities of the positive and the negative charges. Under minute density fluctuation in the volume density of one type of charges at some point, the domain of all other like charges within radius $r_{-}$are trapped gravitationally into a cluster. We will call this domain a daemon (for dark electric matter object). There will be no charges of this type between $r_{-}$and $r_{+}$, while charges of this type on the outside of $r_{+}$would be strongly repelled. As a result, the pre-inflationary Universe nucleates into such domains (daemons). Domains of different charge can, obviously, overlap: an oppositely charged particle, approaching a daemon, will not experience turning radii, will fly into the daemon and freely interact with the particles in it. As our aim is to give a qualitative description, we also assume that $m$ is the typical mass of an elementary particle, while $q$ is its typical charge.

It should be noted that when both turning radii are present, they are always real, that is, that they are real for any value of $\epsilon$ (or any temperature). The discriminant $\left(4 Q^{2} / M^{2}\right)\left(1-q^{2} / m^{2}\right)\left(Q^{2} / M^{2}-1\right)$ of the quadratic expression in $\epsilon$ under the root must then be negative and, indeed, it always is - for all charged elementary particles, $q / m \gg 1$. Also, an arbitrary accumulation of elementary particles of like charge, trapped by the Reissner-Nordström field, necessarily leads to $|Q|>M$. A daemon is, therefore, a naked singularity.

We now turn to the study of quantum tunneling of trapped particles through the classically forbidden region between the two turning radii $r_{ \pm}$. The Schrödinger equa- 
tion of one-dimensional motion along the $r$-axis in potential $(3)$ is: $\psi^{\prime \prime}(r)+\left(A r^{-2}-\right.$ $\left.B r^{-1}\right) \psi(r)=-\left(2 m E / \hbar^{2}\right) \psi(r)$, where $A=\left(2 m / \hbar^{2}\right) a=-m Q^{2}\left(1-q^{2} / m^{2}\right) / \hbar^{2}=$ const $>0$ and $B=\left(2 m / \hbar^{2}\right) b=-2 m M[1-(q Q \epsilon) /(m M)] / \hbar^{2}>0$.

We are interested in the continuum of scattering states $(E>0)$. Using the Wentzel-Kramers-Brillouin (WKB) approximation method (see, ${ }^{9}$ for example), we will determine the transmission coefficient for tunneling through the classically forbidden region between the two turning radii $r_{ \pm}$. The picture is very similar to the Gamow theory of alpha-decay ( see $^{9}$ again).

The Schrödinger equation can be re-written as $\psi^{\prime \prime}(r)=-\left(p^{2} / \hbar^{2}\right) \psi(r)$ where $p(r)=\sqrt{2 m[E-U(r)]}$ is the classical momentum of a particle with energy $E$ moving in potential $U(r)$ (with $E>U(r)$, so that $p(r)$ is real). For tunneling through a potential barrier (namely, across the classically forbidden region between the two turning radii $r_{ \pm}$), the WKB-approximated wave function is given by:

$$
\psi(r) \simeq \frac{D}{\sqrt{|p(r)|}} e^{ \pm \frac{i}{\hbar} \int_{r_{-}}^{r_{+}}|p(r)| d r}
$$

where $D=$ const and $|p(r)|=\sqrt{2 m[U(r)-E]}$.

The amplitude of the transmitted wave, relative to the amplitude of the incident wave, is diminished by the factor $e^{2 \gamma}$, where

$$
\gamma=\frac{1}{\hbar} \int_{r_{-}}^{r_{+}}|p(r)| d r=-\frac{\pi}{\hbar} \sqrt{m}|Q| \sqrt{\frac{q^{2}}{m^{2}}-1}+\frac{\pi}{\hbar} \sqrt{m} M \frac{\epsilon \frac{q}{m} \frac{Q}{M}-1}{\sqrt{\epsilon^{2}-1}} .
$$

The tunneling probability $P$ is proportional to the Gamow factor $e^{-2 \gamma} \cdot{ }^{9}$

With the drop of the temperature (that is, when $\epsilon$ starts falling from $\infty$ towards 1), the inner turning radius $r_{-}$tends to a finite value, while the outer turning radius $r_{+}$tends to infinity. The width of the forbidden classical region, $\delta=r_{+}-r_{-}$, also tends to infinity in the limit $\epsilon \rightarrow 1$ : In the very early Universe, at extremely high temperatures (regime $\epsilon \gg 1$ ), the two turning radii are approximated by $r_{ \pm}=(q Q \pm m|Q|) /(k T)$ and $\gamma$ is not temperature-sensitive: $\left.\gamma\right|_{\epsilon \gg 1}=-(\pi / \hbar) \sqrt{m}|Q|\left(q^{2} / m^{2}-1\right)^{1 / 2}+(\pi q Q) /(\hbar \sqrt{m})$. As the emitted particles have charge with the same sign as that of the daemon, the absolute value $|Q|$ of the total charge of the daemon diminishes. The mass $M$ of the daemon diminishes as well with each emission, but $|Q| / M \simeq$ const $>1$ at all times. Note that we disregard the small effect of re-capture of ejectiles on the rate of decrease of $|Q|$ and $M$. We next expand the first term on the right-hand-side of the last equation up to first order over the small parameter $m / q$. This gives the probability for tunneling $P$ as proportional to $\exp [-(\pi / \hbar) m \sqrt{m}|Q| /|q|]$ in the very early Universe (regime $\epsilon \gg 1$ ) and growing exponentially with the decrease of $|Q|$. Over (dimensionless) time $d t$, the charge of the daemon will decrease by the amount $d|Q|$ proportional to $-P d t$ and, therefore, in the very early Universe, $|Q(t)| \simeq \ln (C-t)$, where $C=$ const and $t$ is dimensionless time. This gives $P(t) \simeq 1 /(C-t)$. In alpha-decay, the daughter nucleus recoils after the emission. In view of the analogies between alpha-decay 
and the current case, we make the following assumption. A particle of kinetic energy $E$ inside the daemon, tunnels through. Tunneling in itself does not change the energy of the particle (otherwise, it would "resurface" at point different from the outer turning radius). The recoil energy (needed for conservation of momentum) however, does: the particle's kinetic energy after the emission will be $E$, diminished by the recoil kinetic energy $E_{R}$. The relativistically correct relation between the linear momentum $p$ of particle of rest mass $m$ and the kinetic energy $E$ of the particle is given by: ${ }^{10} p^{2}=2 m E+4 E^{2} / c^{2}$ and the recoil kinetic energy $E_{R}$ is: ${ }^{10} E_{R}=(m / M) E+2\left(1-m^{2} / M^{2}\right) E^{2} / M$ where $M$ is the mass of the daughter nucleus. Let us first disregard the relativistic (quadratic in $E$ ) corrections. Then, if $E_{0}$ is the total kinetic energy of all particles inside the daemon before the first emission and if we denote $M / m$ by $n$ (figuratively, we have $n$ "equivalent" ingredients inside the daemon), then after the first emission, the ejectile will have energy $E_{1}=E_{0} / n-E_{R}^{(1)}$, where $E_{R}^{(1)}=E_{1} m /(M-m)=E_{1} /(n-1)$. The energy of the first ejectile is therefore $E_{1}=E_{0}(n-1) / n^{2}$. At the same time, as a result of the loss of $m / M$ of the daemon, the total kinetic energy inside the daemon is decreased from $E_{0}$ to $E_{0}-E_{0} / n=E_{0}(n-1) / n$. The second ejectile will have $1 /(n-1)$ of this energy, or energy $E_{0} / n$ prior to leaving the daemon. After tunneling, its energy $E_{2}$ will be $E_{0} / n-E_{R}^{(2)}$, where $E_{R}^{(2)}=E_{2} m /(M-2 m)=E_{2} /(n-2)$. Thus $E_{2}=$ $E_{0}(n-2) /[n(n-1)]$. The energy inside the daemon is decreased from $E_{0}(n-1) / n$ to $E_{0}(n-2) / n$. The energy of the third ejectile prior to leaving the daemon will be $1 /(n-2)$ of the inside energy, or $E_{0} / n$. Thus the energy carried away by the third ejectile will be $E_{3}=E_{0}(n-3) /[n(n-2)]$. The $k^{\text {th }}$ projectile will therefore have energy $E_{k}=E_{0}(n-k) /[n(n-k+1)]=E_{0}(m / M)[1-1 /(M / m-k+1)]$. We now take a continuum limit and re-write this as $E(t)=E_{0} m / M-E_{0} /[(m / M)(m / M-k(t)+1)]$, where $k(t)$ is the number of particles emitted after time $t$. The charge inside a daemon decreses in time from its initial value $Q_{0}$ as $Q(t)=Q_{0}-k(t) q$. Thus $k(t)=Q_{0} / q-(1 / q) \ln (C-t) \simeq M / m-(1 / q) \ln (C-t)$. This gives $E(t) \simeq$ $E_{0}(m / M)\{1-1 /[1+(1 / q) \ln (C-t)]\}$. The temperature drops at least as square root of $E(t)$. The outer turning radius $r_{+}$(which is inversely proportional to the temperature) has an accelerated increase with time. In result, the scale factor of the Universe, $a(t)$, which is proportional to $r_{+}$and, therefore, inversely proportional to the temperature grows with time. The second derivative of $a(t)$ is positive. Therefore we have inflation. If the relativistic corrections,${ }^{10}$ mentioned earlier, were included, than the rapid drop in $T$ would be even more pronounced.

Note that in the regime $\epsilon \gg 1$, the width $r_{+}-r_{-}=2 m|Q| /(k T)$ of the classically forbidden region initially even decreases with time (as the drop of the temperature $T$ is not, initially, as fast as the drop of the charge $|Q|$ of the daemon - tunneling is practically temperature-independent). This is when huge amounts of particles gush out of the daemons. The extremely rapid drop in the temperature that follows leads to an extremely rapid growth of $r_{+}$, together with that of $a(t)$. The "graceful exit" of the inflation occurs when the width $r_{+}-r_{-}=2 m|Q| /(k T)$ of the barrier grows large enough so that quantum tunneling is switched off. This happens before 
daemons become fully depleted (bound states inside the daemons should also not be forgotten). In other words, when the temperature $T$ drops sufficiently and the second term in expression (6) for $\gamma$, namely $(\pi / \hbar) M \sqrt{m}[\epsilon q Q /(m M)-1]\left(\epsilon^{2}-1\right)^{-1 / 2}$, takes control, a break is put on the tunneling (the lower limit of this term is $(\pi / \hbar) q Q / \sqrt{m}$ when $\epsilon \gg 1$ ). As the probability for tunneling is brought down very rapidly towards 0 and particles are no longer ejected by the daemons, the medium outside the daemons is no longer cooled by the tunneling process. Without quantum tunneling, the charges of the daemons remain practically constant. However, the temperature of the outside fraction of the Universe continues to drop after the rapid accelerated expansion as a different expansion mechanism has naturally taken over. This is the recently proposed Reissner-Nordström expansion mechanism: ${ }^{5,6}$ with constant charges of daemons, the Universe continues to cool: $T \simeq t^{-1 / 2}$, and expand: $a \simeq t^{1 / 2}$. This is the start of the radiation dominated epoch. It is also characterized as the beginning of a supercooling phase. At the end of the inflation, the daemons are still much hotter than the outside fraction of the Universe. A daemon will now cool not through quantum tunneling, but through interaction with the particles of oppositely charged daemons, which, in turn interact with the particles outside the original daemon. In view of the low densities, this does not happen as fast as the Universe expands. Eventually, the temperature of the daemons and the temperature of the "free" fraction of the Universe will equalize and, in result, the Universe will have reheated, but not enough to reignite the inflation (as the daemon temperature now is lower than the one at the end of the inflation and quantum tunneling cannot start). During the reheating, the scale factor $a(t)$ of the Universe does not decrease as there is no mechanism to draw particles, blown away by the growth of the daemons' outer radii, back towards the daemons: the decrease in the outer turning radius $r_{+}$ of a daemon simply means that particles of the outer fraction will penetrate deeper and deeper into the repulsive field of the daemons. The Universe then enters into another supercooling phase followed by another reheating. This process is repeated until daemons cool down to the temperature of the surrounding fraction and cannot re-ignite futher reheatings. Then the temperature drop will simply follow $T \simeq t^{-1 / 2}$ and the expansion will be at the rate of $\sqrt{t}$.

\section{References}

1. S. Hawking, Mon. Not. R. Astr. Soc. 152, 75-78 (1971).

2. H. Reissner, Ann. Phys. (Germany) 50, 106-120 (1916);

G. Nordström, Proc. Kon. Ned. Akad. Wet. 20, 1238-1245 (1918).

3. C.W. Misner, K.S. Thorne and J. Wheeler, Gravitation, W.H. Freeman (1973).

4. R.H. Boyer and R.W. Lindquist, J. Math. Phys. 8 (2), 265 (1967).

5. E.M. Prodanov, R.I. Ivanov, and V.G. Gueorguiev, Astroparticle Physics 27 (150-154) 2007, hep-th/0703005.

6. E.M. Prodanov, R.I. Ivanov, and V.G. Gueorguiev, Journal of High Energy Physics, JHEP 06(2008)060, arXiv: 0608.0076.

7. J.M. Cohen and R. Gautreau, Phys. Rev. D 19 (8), 2273-2279 (1979).

8. R.M. Wald, General Relativity, University of Chicago Press (1984). 
9. D.J. Griffiths, Introduction to Quantum Mechanics, Pearson Prentice Hall (2005).

10. M. Stanley Livingston and H.A. Bethe, Rev. Mod. Phys. 9(3), 245-390 (1937). 